\title{
CRÓNICA DE LAS XXII JORNADAS DE LA SOCIEDAD ESPAÑOLA DE FILOSOFÍA JURÍDICA Y POLÍTICA, «VIEJOS TEMAS, NUEVOS PROBLEMAS ». UNIVERSIDAD DE LA RIOJA, LOGROÑo, 26 Y 27 DE MARZO DE 2009
}

\author{
Raúl SUSÍN BETRÁN \\ Profesor Titular de Filosofía DEL DERECHO \\ UNIVERSIDAD DE LA RIOJA
}

A finales del mes de marzo de 2009 , los días 26 y 27, y tal y como se había acordado en la Asamblea de la Sociedad Española de Filosofía Jurídica y Política que tuvo lugar en la Universidad de Alcalá de Henares en marzo de 2007, se celebraron en la Universidad de La Rioja, Logroño, las sesiones de las XXII Jornadas de nuestra Sociedad. En esta ocasión, se vertebraron en torno al título «Viejos temas, nuevos problemas», en un intento de hacer alusión a una propuesta de desarrollar temas «clásicos» a través de cuestiones actuales, y la organización corrió a cargo del área de Filosofía de Derecho de la Universidad anfitriona.

En sintonía con la dirección que tomaron las Jornadas desde 2007, las celebradas en la Universidad de Alcalá, se pretendió que la estructura fuera algo diferente a la que se considera más tradicional. De esta forma, realizadas una serie de consultas a los miembros del comité asesor y a la misma dirección de la Sociedad, desde la Universidad de La Rioja diseñamos una dinámica de trabajo que cumpliera, de un lado, con ese interés de renovación; y de otro, recogiera, en la medida de lo posible, las distintas sensibilidades y opciones académicas que integran la Sociedad. Así, el programa quedó configurado con tres sesiones de exposición de ponencias y tres talleres-seminarios, además de un tiempo para la defensa de una selección de las comunicaciones recibidas, y al margen de una serie de actividades complementarias fuera de lo estrictamente académico, todo ello puntualmente anunciado en una página web que se habilitó al efecto.

El primer día, el jueves 26 de marzo, y tras el proceso de bienvenida, acreditación y entrega de material, tuvo lugar el acto de inauguración en el Aula Magna de la Universidad, donde se desarrollaron todas las Jornadas, y que contó con la presencia del Consejero de Educación, Cultura y Deportes del Gobierno de La Rioja, el Rector de la Universidad de La Rioja y el Decano de la Facultad de Ciencias Jurídicas y Sociales, además, lógicamente, de representantes de la Junta Directiva de la Sociedad y de la Organización de las Jornadas. Inmediatamente después dio comienzo la primera de las tres sesiones de ponencias. Cada una de estas tres sesiones contó con tres intervenciones de unos 20-25 minutos cada una, y con una persona que actuó como presidente-moderador, que realizó una presentación general de la sesión y posteriormente hizo una recapitulación de las 
cuestiones que consideró más sugerentes, dando pie, en cada caso, a interesantes debates. La primera sesión llevaba por título «Derechos Humanos. Algunos retos actuales» y en ella se tocaba un tema que ha sido de interés para buena parte de los miembros de la Sociedad. La presidencia de la Sesión corrió a cargo de Jesús Ignacio Martínez García de la Universidad de Cantabria, y en ella intervinieron, por este orden, María José Añón Roig, Universidad de Valencia, con la ponencia «Una propuesta para garantizar el derecho a la existencia: la renta básica»; Pablo de Lora Deltoro, Universidad Autónoma de Madrid, con «La familia humana y otros animales»; y Antonio Madrid Pérez, Universidad de Barcelona, con una intervención titulada «El acceso a los derechos: las experiencias del proyecto dret al Dret».

Tras una pausa para un descanso y un café continuaron las Jornadas con la segunda de las sesiones, «Derecho-Política-Poder», otra de las cuestiones que han estado presentes en los trabajos de numerosos miembros de la Sociedad. En este caso, las tres intervenciones fueron, por este orden: Macario Alemany García, Universidad de Alicante, «Paternalismo y democracia»; Encarna Bodelón González, Universidad Autónoma de Barcelona, «Leyes de igualdad y derechos con perspectiva de género»; y Alfonso Ruiz Miguel, Universidad Autónoma de Madrid, «Educación ciudadana y neutralidad estatal»; y presidiendo y moderando la sesión y el debate, José Ignacio Lacasta Zabalza de la Universidad de Zaragoza. Al finalizar esta segunda sesión tuvo lugar una comida para todos los participantes de las Jornadas en la misma Universidad de La Rioja.

Se reanudaron las Jornadas a primera hora de la tarde, con un tiempo dedicado a la presentación y defensa de una selección de las comunicaciones recibidas y donde se pudo observar una variedad de temas que cabe ser leída como una buena salud de la diversidad de intereses entre los miembros de la Sociedad. A continuación tuvo lugar el primero de los seminarios, el dedicado a Cine $y$ Derecho y que como muy correctamente dijo uno de sus coordinadores, Benjamín Rivaya García, de la Universidad de Oviedo, suponía una «puesta de largo» de esta relación, cine y Derecho, que ha dado lugar a asignaturas, seminarios, proyectos de investigación e, incluso, a una colección bibliográfica que dirige otro de los que fueron responsables de este seminario, Javier de Lucas Martín, Universidad de Valencia. El seminario se inició con una selección de escenas vinculadas con el cine y el Derecho preparada desde la Organización. Siguió una ponencia del ya citado Benjamín Rivaya, «Las posibilidades del cine para la docencia e investigación jurídica»; y a continuación hubo una mesa redonda en las que, de forma práctica y a través de una serie de breves proyecciones, se expusieron distintas experiencias y propuestas de utilización del cine como herramienta docente y de investigación jurídica. En dicha mesa redonda participaron: Ricardo García Manrique, Universidad de Barcelona, José Luis Pérez Triviño, Universidad Pomepu Fabra de Barcelona, María José García Salgado, Universidad de Oviedo, Mario Ruiz Sanz, Universidad de Valencia, y Juan Antonio Gómez García, Universidad Nacional de Educación a Distancia. Bien entrada la tarde concluía este seminario en el que no sólo se habló de cine y Derecho y de su relación; sino donde se expusieron, a la vez, cuestiones de investigación y de docencia, lo que, entendimos, contribuyó a valorizar aún más la que fue una de las actividades que contó con un mayor eco entre los asistentes. Finalmente, y ya en la noche del día 26 , tuvo lugar, como actividad complementaria a lo académico, una visita nocturna guiada por el Logroño histórico.

La jornada del viernes 27 de marzo se inició con la «tercera sesión», la dedicada a «Derecho y Ciencia: Cuestiones de bioética y biotecnología». Presidida y moderada por Andrés Ollero Tassara, Universidad Rey Juan Carlos, esta sesión contó con las intervenciones siguientes: Ascensión Cambrón Infante, Universidad de A Coruña, expuso «Los problemas de la biotecnología y su tratamiento jurídico. ¿Ciencia o doctrina del Derecho?»; Héctor Silveira Gorski, Universidad de Lleida, «El derecho ante la tecnociencia genética. Reflexiones y desafíos»; y Ángel Pelayo González-Torre, Universidad de Cantabria, «La autonomía personal en el seno de la relación médica. El consentimiento informado». Tras el debate sobre las cuestiones suscitadas en esta sesión hubo un descanso-café, y 
posteriormente se desarrollaron el segundo y tercer seminario, «coloquio sobre un libro con su autor». Algún pequeño cambio de última hora hizo conveniente una reestructuración de la dinámica de estos dos seminarios, que habían sido pensados para realizarse simultáneamente en dos espacios más reducidos. Al final, se unificaron los dos seminarios, lo que no quitó interés a los mismos. Resultó un seminario presidido por José Martínez de Pisón Cavero, Universidad de La Rioja, y en él se discutió sobre dos libros con sus dos autores. El presidente de la Sociedad, Carlos Alarcón Cabrera, Universidad Pablo de Olavide de Sevilla, fue el encargado de debatir con Francisco Laporta San Miguel, Universidad Autónoma de Madrid, sobre el libro de este último El imperio de la Ley. Una visión actual. Mientras que Manuel Calvo García, Universidad de Zaragoza, se ocupó de debatir con Jorge Malem Seña, Universidad Pompeu Fabra, a propósito del libro de éste titulado, El error judicial y la formación de los jueces. Esta actividad, que no es extraña en congresos y jornadas de otras disciplinas, fue pensada como una vía para fomentar la discusión y el debate entre los asistentes. En la elección de las publicaciones se atendió tanto a unos criterios de cercanía temporal, como de que pudieran ser los temas de un interés general y de actualidad. La dinámica, la que se había pensado, en grupos más reducidos, o la que se hizo finalmente, con todos los asistentes, puede, sin duda, mejorarse, como también pueden mejorarse los criterios de selección de las publicaciones; pero creemos que, con todo, quedó una imagen positiva de la actividad y que se podría repetir en sucesivas Jornadas. Por otra parte, no se puede dejar de lado que ese mismo mes, marzo de 2009, se había fallado el Premio Nacional de Investigación «Pascual Madoz» en el área de Derecho y Ciencias Económicas y Sociales en el autor del primero de los libros, Francisco Laporta.

Las Jornadas se cerraron con la celebración de la Asamblea de la Sociedad, donde se aceptó la propuesta de los colegas de la Universidad de Extremadura de responsabilizarse de la organización de las XXIII Jornadas. Ya para acabar nos desplazamos a la Bodega Dinastía Vivanco, Briones, La Rioja, donde tuvo lugar una comida y una posterior visita al Museo Cultura del Vino sito en la referida bodega. 